Homology, Homotopy and Applications, vol.18(2), 2016, pp.177-181

\title{
KHOVANOV HOMOTOPY TYPES AND THE DOLD-THOM FUNCTOR
}

\author{
BRENT EVERITT, ROBERT LIPSHITZ, SUCHARIT SARKAR AND \\ PAUL TURNER \\ (communicated by Ralph Cohen)
}

\begin{abstract}
We show that the spectrum constructed by Everitt and Turner as a possible Khovanov homotopy type is a product of Eilenberg-MacLane spaces and is thus determined by Khovanov homology. By using the Dold-Thom functor it can therefore be obtained from the Khovanov homotopy type constructed by Lipshitz and Sarkar.
\end{abstract}

A Khovanov homotopy type is a way of associating a (stable) space to each link $L$ so that the classical invariants of the space yield the Khovanov homology of $L$. There are two recent constructions of Khovanov homotopy types, using different techniques and giving different results $[\mathbf{E T 1 4}, \mathbf{L S 1 4 a}]$. In [ET14] homotopy limits were employed to build an $\Omega$-spectrum $\mathbf{X}_{\bullet} L=\left\{X_{k}(L)\right\}$ with the following properties:

1. The homotopy type is a link invariant, and

2. the homotopy groups are Khovanov homology:

$$
\pi_{i}\left(\mathbf{X}_{\bullet}(L)\right)=K h^{-i}(L) .
$$

The goal of this note is to prove the following result:

Main Theorem. Each of the spaces $X_{k}(L)$ is homotopy equivalent to a product of Eilenberg-MacLane spaces.

In $[\mathbf{L S 1 4 a}]$ the programme of Cohen, Jones and Segal [CJS95] was generalized to produce a suspension spectrum $\mathcal{X}_{K h}(L)$ with the following properties:

1. The homotopy type is a link invariant, and

2. the reduced cohomology is Khovanov homology:

$$
\widetilde{H}^{i}\left(\mathcal{X}_{K h}(L)\right)=K h^{i}(L) .
$$

As a corollary we obtain that $\mathbf{X}_{\bullet}(L)$ is homotopy equivalent to the infinite symmetric product of $\mathcal{X}_{K h}(L)$.

The second author was supported by NSF grant number DMS-0905796 and a Sloan Research Fellowship. The third author was supported by a Clay Mathematics Institute Postdoctoral Fellowship. Received December 6, 2014, revised June 1, 2015; published on September 14, 2016. 2010 Mathematics Subject Classification: 57M25, 55P42.

Key words and phrases: Khovanov homology, spectra, Dold-Kan correspondence.

Article available at http://dx.doi.org/10.4310/HHA.2016.v18.n2.a9

Copyright (C) 2016, International Press. Permission to copy for private use granted. 
To prove Theorem 1 we use an explicit model, due to McCord [McC69], of the Eilenberg-MacLane spaces. Given a monoid $G$ and a based topological space $X$, let $B(G, X)$ denote the set of maps $u: X \rightarrow G$ such that $u(x)=0$ for all but finitely many $x \in X$. Then $B(G, X)$ is a monoid, and if $G$ is a group (the case of interest) then $B(G, X)$ is a group. Moreover, when $G$ is an abelian topological group the set $B(G, X)$ can be topologized in a natural way so that the group operation is continuous. This construction has nice functoriality: letting $\mathrm{Ab}, \mathrm{Top}_{*}$ and AbTop denote, respectively, the categories of abelian groups, based topological spaces and topological abelian groups, one has the following result [McC69, Proposition 6.7]:

Proposition 1. McCord's construction is a bifunctor

$$
B(-,-): \mathrm{Ab} \times \mathrm{Top}_{*} \rightarrow \text { AbTop. }
$$

Furthermore, as a special case of [McC69, Theorem 11.4], for an abelian group $G$ the space $B\left(G, S^{n}\right)$ is the Eilenberg-MacLane space $K(G, n)$. Thus we may take as the Eilenberg-MacLane space functor:

$$
B\left(-, S^{n}\right): \text { Ab } \rightarrow \text { AbTop. }
$$

Conversely, the following is [Hat02, Corollary 4K.7, p. 483] (apparently originally due to Moore; cf. [McC69, p. 295]):

Proposition 2. A path-connected, commutative topological monoid is a product of Eilenberg-MacLane spaces.

The spaces $X_{k}(L)$ are built as homotopy limits of diagrams of spaces. Recall that given a small category $\mathrm{C}$ and a (covariant) functor $D: \mathrm{C} \rightarrow \mathrm{Top}_{*}$ (a diagram), that $\operatorname{holim}_{\mathrm{C}} D$ is constructed as follows (see, e.g., [BK72, Section 11.5]). Consider the product

$$
\prod_{\sigma \in N(\mathrm{C})} \operatorname{Hom}\left(\Delta^{n}, D\left(c_{n}\right)\right)=\prod_{n \geqslant 0} \prod_{c_{0} \rightarrow \cdots \rightarrow c_{n}} \operatorname{Hom}\left(\Delta^{n}, D\left(c_{n}\right)\right),
$$

where $N(\mathrm{C})$ is the nerve of $\mathrm{C}$ consisting of all sequences of composable morphisms $\sigma=\left(c_{0} \stackrel{\alpha_{1}}{\longrightarrow} c_{1} \stackrel{\alpha_{2}}{\longrightarrow} \cdots \stackrel{\alpha_{n}}{\longrightarrow} c_{n}\right)$ and Hom denotes the space of continuous maps from the standard $n$-simplex. The homotopy $\operatorname{limit}_{\text {holim }} D$ is the subspace of this product consisting of those tuples $\left(f_{\sigma}\right)_{\sigma \in N(\mathrm{C})}$ such that the following diagrams commute:
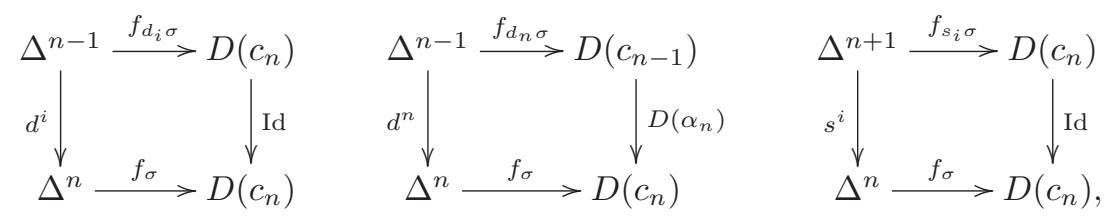

with $0 \leqslant i<n$ on the left and $0 \leqslant i \leqslant n$ on the right. Here the $d^{i}$ and $s^{i}$ are coface and codegeneracy maps and $d_{i}$ and $s_{i}$ are the face and degeneracy maps of the nerve 
given by

$$
d_{i} \sigma=\left(c_{0} \stackrel{\alpha_{1}}{\longrightarrow} \cdots c_{i-1} \stackrel{\alpha_{i+1} \alpha_{i}}{\longrightarrow} c_{i+1} \cdots \stackrel{\alpha_{n}}{\longrightarrow} c_{n}\right)
$$

and

$$
s_{i} \sigma=\left(c_{0} \stackrel{\alpha_{1}}{\longrightarrow} \cdots c_{i} \stackrel{\mathrm{Id}}{\longrightarrow} c_{i} \cdots \stackrel{\alpha_{n}}{\longrightarrow} c_{n}\right),
$$

with $d_{0}, d_{n}$ similarly.

The following is well-known, but for completeness we give its (short) proof.

Proposition 3. Let $D: C \rightarrow \mathrm{Top}_{*}$ be a diagram of topological abelian groups and continuous group homomorphisms. Then the homotopy limit of $D$ is a topological abelian group.

Proof. Pointwise addition makes the set $\operatorname{Hom}\left(\Delta^{n}, D\left(c_{n}\right)\right)$ into an abelian group, and the product in formula (1) is the product (topological abelian) group. It remains to see that the diagrams (2) describe a subgroup of this product. Suppose that tuples $\left(f_{\sigma}\right)$ and $\left(g_{\sigma}\right)$ make these diagrams commute. Then the first and last diagrams automatically commute for the pointwise sum $\left(f_{\sigma}+g_{\sigma}\right)$. The middle diagram for the pointwise sum becomes,

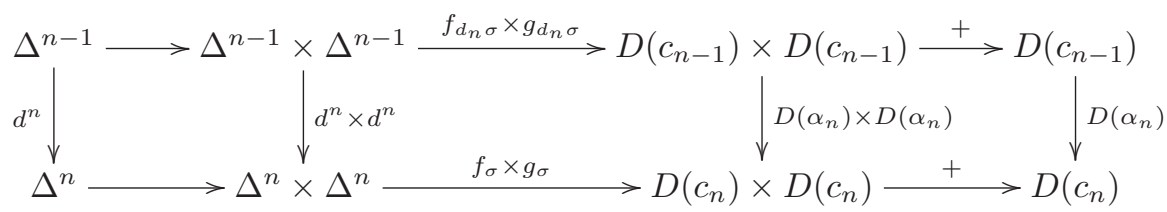

for which the first square obviously commutes, the second commutes since $f$ and $g$ are in the prescribed subspace and the third commutes from the fact that $D\left(\alpha_{n}\right)$ is a group homomorphism. The inverse operation is similarly seen to be closed, hence the subspace defined above is a subgroup.

We are now ready for the main theorem:

Theorem 1. Each of the spaces $X_{k}(L)$ is homotopy equivalent to a product of Eilenberg-MacLane spaces.

Proof. Let $L$ be an oriented link diagram with $c$ negative crossings. The space $X_{k}(L)$ is constructed as follows. Let $I$ denote the category with objects $\{0,1\}$ and a single morphism from 0 to 1 , and $I^{n}$ the product of $I$ with itself $n$ times. Let $\overline{0}$ be the initial object in $I^{n}$, and let $\mathbf{P}$ be the result of adjoining one more object to $I^{n}$ and a single morphism from the new object to every object except $\overline{0}$.

In $[\mathbf{E T 1 4}]$ it is shown that there is a functor $F: \mathbf{P} \rightarrow \mathrm{Ab}$ such that the $i$ th derived functor of the inverse limit, $\lim _{\mathbf{P}}{ }^{i} F$, is isomorphic to the $i$ th unreduced Khovanov homology of $L$. The space $X_{k}(L)$ is constructed by composing this functor with the Eilenberg-MacLane space functor $K(-, k+c)$ and taking the homotopy limit of the resulting diagram of spaces.

We may now use the explicit model for Eilenberg-MacLane spaces given by McCord. By applying Proposition 1 we define a diagram $D: \mathbf{P} \rightarrow$ AbTop as the composition $B\left(-, S^{k+c}\right) \circ F: \mathbf{P} \rightarrow \mathrm{Ab} \rightarrow$ AbTop. By the homotopy invariance property 
of the homotopy limit construction we have

$$
X_{k}(L) \simeq \operatorname{holim}_{\mathbf{P}} D .
$$

By Proposition 3, the homotopy limit on the right is itself a topological abelian group, and hence, by Proposition 2, a product of Eilenberg-MacLane spaces.

Corollary 1. The homotopy type of $\mathbf{X}_{\bullet}(L)$ is determined by $K h(L)$.

The spectrum $\mathcal{X}_{K h}(L)=\left\{\mathcal{X}_{K h}^{(k)}(L)\right\}$ constructed in $[\mathbf{L S 1 4 a}]$ has the additional property that the cellular cochain complex of the space $\mathcal{X}_{K h}^{(k)}(L)$ is isomorphic to the Khovanov complex of $L$ (up to shift). It follows from the description of the Khovanov homology of the mirror image (see $[\mathbf{K h o 0 0}]$ ) that

$$
\widetilde{H}_{i}\left(\mathcal{X}_{K h}(L)\right)=K h^{-i}(-L)
$$

where $-L$ denotes the mirror of $L$. The infinite symmetric product $\operatorname{Sym}^{\infty} \mathcal{X}_{K h}^{(k)}(L)$ is seen from the Dold-Thom theorem to be

$$
\operatorname{Sym}^{\infty} \mathcal{X}_{K h}^{(k)}(L)=\prod_{n} K\left(\widetilde{H}_{n}\left(\mathcal{X}_{K h}^{(k)}(L)\right), n\right)
$$

from which we have the following:

Corollary 2. For large enough $k$, the space $X_{k}(-L)$ is homotopy equivalent to the infinite symmetric product $\operatorname{Sym}^{\infty} \mathcal{X}_{K h}^{(k)}(L)$.

We end by noting that the analogue of Theorem 1 for the spectra $\mathcal{X}_{K h}(L)$ is not true. For all alternating knots $\mathcal{X}_{K h}(L)$ is a wedge of Moore spaces $[\mathbf{L S 1 4 a}]$, however there are examples of non-alternating knots for which $\mathcal{X}_{K h}(L)$ is not a wedge of Moore spaces (see $[\mathbf{L S 1 4 b}]$ ).

\section{Acknowledgements}

We thank Tyler Lawson for several helpful suggestions, including communicating Proposition 3 to us.

\section{References}

[BK72] A.K. Bousfield and D.M. Kan, Homotopy Limits, Completions and Localizations, Lecture Notes in Math., vol. 304, Springer-Verlag, Berlin, 1972.

[CJS95] R.L. Cohen, J.D.S. Jones, and G.B. Segal, Floer's infinite-dimensional Morse theory and homotopy theory, The Floer Memorial Volume, Progr. Math., vol. 133, Birkhäuser, Basel, 1995, pp. 297-325.

[ET14] B. Everitt and P. Turner, The homotopy theory of Khovanov homology, Algebr. Geom. Topol. 14 (2014), 2747-2781.

[Hat02] A. Hatcher, Algebraic Topology, Cambridge University Press, Cambridge, 2002 .

[Kho00] M. Khovanov, A categorification of the Jones polynomial, Duke Math. J. 101 (2000), no. 3, 359-426. 
[LS14a] R. Lipshitz and S. Sarkar, A Khovanov stable homotopy type, J. Amer. Math. Soc. 27 (2014), no. 3, 983-1042.

[LS14b] R. Lipshitz and S. Sarkar, A Steenrod square on Khovanov homology, J. Topol. 7 (2014), no. 3, 817-848.

[McC69] M.C. McCord, Classifying spaces and infinite symmetric products, Trans. Amer. Math. Soc. 146 (1969), 273-298.

Brent Everitt brent.everitt@york.ac.uk

Department of Mathematics, University of York, York, YO10 5DD, UK

Robert Lipshitz lipshitz@uoregon.edu

Department of Mathematics, University of Oregon, Eugene, OR 97403, USA

Sucharit Sarkar sucharit@math.ucla.edu

Department of Mathematics, University of California, Los Angeles, CA 90095 USA

Paul Turner Paul.Turner@unige.ch

Section de mathématiques, Université de Genève, 2-4 rue du Lièvre, CH-1211, Geneva, Switzerland 